Rech. Transp. Secur.

http://www.necplus.eu/RTS

Additional services for Rech. Transp. Secur.:

Email alerts: $\underline{\text { Click here }}$

Subscriptions: $\underline{\text { Click here }}$

Commercial reprints: Click here

Terms of use : $\underline{\text { Click here }}$

\title{
Améliorer la sécurité des piétons des quartiers défavorisés : l'expérience britannique d'une approche socio-spatiale de la sécurité routière
}

Sylvanie Godillon

Rech. Transp. Secur. / Volume 2014 / Issue 01 / March 2014, pp 21 - 36

DOI: 10.4074/S0761898014001022, Published online: 11 June 2014

Link to this article: http://www.necplus.eu/abstract S0761898014001022

How to cite this article:

Sylvanie Godillon (2014). Améliorer la sécurité des piétons des quartiers défavorisés : l'expérience britannique d'une approche socio-spatiale de la sécurité routière. Rech. Transp. Secur., 2014, pp 21-36 doi:10.4074/S0761898014001022

Request Permissions : $\underline{\text { Click here }}$ 


\title{
Améliorer la sécurité des piétons des quartiers défavorisés : l'expérience britannique d'une approche socio-spatiale de la sécurité routière
}

\author{
Improving the traffic safety of pedestrians in deprived neighbourhoods: \\ Case-study of British socio-spatial approach of road safety
}

\author{
Sylvanie Godillon
}

Reçu le 30 novembre 2012, accepté le 14 janvier 2014

(C) IFSTTAR et Éditions NecPlus 2014

\begin{abstract}
Résumé Si la sécurité du piéton dans les centres urbains s'est améliorée, l'enjeu demeure d'actualité dans les quartiers défavorisés. En effet, le risque d'être impliqué dans un accident est important pour les habitants de quartiers défavorisés en raison d'une forte mobilité piétonne, d'une appropriation des espaces publics et de présence de trafics de transit. Si cette problématique n'est pas appréhendée en France, le Royaume-Uni a expérimenté un fonds spécifique, la Neighbourhood Road Safety Initiative, visant à réduire le nombre de victimes, principalement piétonnes, dans les quartiers défavorisés. Est ainsi expérimenté le traitement d'inégalités socio-spatiales par l'espace. Comment s'opère la transversalité entre les politiques sociales, la rénovation urbaine et les mesures impulsées par la sécurité routière ? L'étude des mises en œuvre locale de ce fonds dans le Grand Manchester permet d'éclairer cette territorialisation de la sécurité routière. L'analyse démontre qu'une gestion centralisée et pragmatique permet de réduire considérablement le nombre d'accidents dans les quartiers pauvres et, précisément, de réduire les risques d'accident pour les piétons.
\end{abstract}

Mots clés sécurité routière · inégalités socio-spatiales · Grand Manchester · rénovation urbaine

\begin{abstract}
While the safety of pedestrians in city centres has increased, the issue is more than ever relevant in deprived neighbourhoods. Indeed, the risk of accident is high for the inhabitants of deprived neighbourhoods because of a high pedestrian mobility, use of public spaces and heavy
\end{abstract}

Sylvanie Godillon $(\bowtie)$

UMR Géographie-Cités (équipe CRIA)

191 rue Saint Jacques, 75005 Paris

e-mail : godillon_sylvanie@yahoo.fr through traffic. While this problematic is not appreciated in France, the United Kingdom has tried out specific funding, the Neighbourhood Road Safety Initiative, aiming at reducing the number of victims, mainly pedestrians, in deprived neighbourhoods. Socio-spatial inequalities are thus handled through a neighbourhood approach. How does the cross-cutting approach operate across social policies, urban renewal and road safety measures? The study of the NRSI in Greater Manchester enables light to be thrown on this territorialisation of road safety. This analysis demonstrates that a centralised and pragmatic management allows accidents in deprived neighbourhoods to be reduce significantly, and specifically risks for pedestrians to be reduce.

Keywords Road safety - Socio-spatial inequalities · Greater Manchester · Urban Renewal

\section{Introduction}

Les espaces publics sont le lieu de multiples usages à la fois dynamiques et statiques. Les aspects dynamiques sont liés au mouvement rapide des véhicules en déplacements alors que les dimensions statiques relèvent de l'ancrage plus local d'un usager lent en déplacement, comme le piéton. Ces deux logiques créent des situations conflictuelles [1]. La multiplicité des fonctions urbaines s'inscrit à l'échelle de la rue et ces différentes logiques peuvent conduire à des conflits, matérialisés notamment par des accidents [2]. Les aménagements d'espaces publics tentent de concilier ces deux dimensions. Après une place fortement accordée à la vitesse en ville, la place du piéton redevient une préoccupation importante dans les choix d'aménagement [3]. Si la sécurité du piéton dans les centres urbains 
s'est améliorée depuis quelques décennies, l'enjeu demeure d'actualité dans les quartiers défavorisés. En effet, le risque d'être impliqué dans un accident est plus fort pour les habitants de quartiers défavorisés ${ }^{1}[4,5,6]$. Les hypothèses explicatives avancées sont une exposition au risque d'accident importante en raison d'une forte mobilité piétonne, ainsi qu'une forte appropriation des espaces publics à proximité du domicile et des quartiers plus traversés par des trafics de transit [7, 8]. Le piéton constitue alors un enjeu important pour réduire les inégalités devant les risques d'accident et améliorer la qualité des déplacements en milieu urbain.

En France, les inégalités devant les risques d'être impliqué dans un accident sont peu connues des pouvoirs publics et ne font pas l'objet d'attention particulière. Or, la désignation des risques comme problème public s'explique comme les résultats d'arbitrages par les pouvoirs publics, comme la confrontation de la société civile avec les pouvoirs publics, et comme les résultats de la manière dont de multiples acteurs définissent et construisent les problèmes [9]. Au contraire, au Royaume-Uni, les nombreuses recherches montrant des inégalités devant l'insécurité des déplacements surtout chez les enfants $[10,11,12]$ ont conduit à l'adoption d'un fonds spécifique à la réduction du nombre d'accidents, la Neighbourhood Road Safety Initiative (NRSI), dans les quartiers défavorisés, avec une forte attention accordée aux enfants piétons. La Neighbourhood Road Safety Initiative (NRSI) est une expérimentation entre 2002 et 2008 qui impulse un traitement des inégalités socio-spatiales devant le risque routier par l'espace : en aménageant les quartiers pour réduire les accidents des piétons, les pouvoirs publics visent à réduire les risques des habitants de ce quartier. Dans quelles mesures des actions spatiales peuvent réduire des inégalités socio-spatiales ? Quelles sont les clés de réussite d'une telle mesure ? L'initiative a permis de réduire considérablement le nombre de tués et de blessés dans les quartiers défavorisés et le nombre d'impliqués parmi les habitants [13]. Les objectifs de cet article sont de comprendre les caractéristiques et la mise en œuvre de ce financement innovant à la frontière entre les actions de la rénovation urbaine et de la sécurité routière, ainsi que d'interroger la dimension innovante de cette politique.

Cette initiative n'a pas été reconduite après son évaluation positive en 2010. L'évolution du contexte financier et politique du Royaume-Uni explique l'absence de cette reconduction. La crise financière de 2009 a conduit à une

\footnotetext{
${ }^{1}$ Les quartiers défavorisés sont définis dans cet article comme des espaces accueillant une part importante d'habitants connaissant des difficultés socio-économiques (chômage, précarité de l'emploi, échec scolaire...). Dans cet article, les quartiers défavorisés correspondent au périmètre institutionnel des politiques de « rénovation urbaine », autrement dit, pour le Royaume-Uni, des quartiers éligibles au fonds du Neighbourhood Renewal.
}

diminution des ressources financières des autorités locales, se traduisant par des réductions budgétaires. Parallèlement, le gouvernement de coalition qui dirige depuis 2010 (Conservateurs et Libéraux-Démocrates) a impulsé une politique moins centralisée, se reposant davantage sur les autorités locales. Il s'agira donc de s'interroger sur la poursuite des pratiques impulsées par la NRSI sans le financement dédié.

Après avoir exposé le contexte et la méthode d'analyse, l'article s'intéressera à la mise en ouvre locale de ce fonds dans le Grand Manchester ${ }^{2}$ afin d'éclairer cet exemple unique de territorialisation de la sécurité routière. Une gestion centralisée et pragmatique permet de réduire considérablement le nombre d'accidents dans les quartiers pauvres et de réduire les risques d'accident pour les piétons, en conduisant des actions ciblées à la fois territoriales et socio-démographiques.

\section{La Neighbourhood Road Safety Initiative pour réduire les accidents dans les quartiers défavorisés}

Des recherches récentes confirment l'influence des caractéristiques du quartier de résidence pour comprendre les inégalités devant le risque d'être impliqué dans un accident. Devant ce constat, la puissance publique britannique a fait le choix d'agir sur les espaces pour réduire ces inégalités sociales. L'analyse du cas du Grand Manchester permet d'éclairer la transversalité entre les actions de rénovation urbaine territorialisée à l'échelle d'un quartier et les mesures pour réduire le nombre de tués et de blessés.

\section{Plus de risque d'accident pour les habitants des quartiers défavorisés}

En dépit d'une amélioration de la sécurité dans les pays industrialisés depuis les années 1970, il existe des inégalités socio-spatiales d'être impliqué dans un accident lors des déplacements. Des travaux de recherche montrent que les habitants des quartiers défavorisés sont plus impliqués dans un accident que des habitants de quartiers aisés $[4,5,6]$. Des travaux récents décomposent l'influence des différentes variables individuelles, familiales et sociales de celles liées aux contextes socio-économiques du quartier de résidence et montrent que les risques d'accident sont plus forts pour les

\footnotetext{
${ }^{2}$ La recherche a été réalisée dans le cadre d'une thèse de doctorat en Géographie - Aménagement à 1'UMR Géographie Cité (équipe CRIA) et à l'Ifsttar (Mécanismes d'Accidents) soutenue en mars 2012. L'auteur tient à remercier les deux laboratoires d'avoir permis le déroulement de cette recherche.
} 
habitants de quartiers pauvres $[14,15,16]$. L'influence du quartier de résidence se combine avec des caractéristiques individuelles [17], des caractéristiques de mobilité [18], des caractéristiques du lieu d'accident [19] et les différentes manifestations de la pauvreté [20].

Dans la mesure où les personnes pauvres ${ }^{3}$ ont 20 à $40 \%$ plus d'accidents que les personnes aisées [21], un effet de composition peut expliquer les risques supplémentaires, autrement dit les habitants des quartiers défavorisés auraient plus d'accidents en raison de leur profil social. En effet, plusieurs travaux se sont centrés sur l'influence des facteurs socio-économiques sur le risque d'avoir un accident : les bas niveaux socio-économiques sont associés à de plus forts taux d'accidents [22]. Les facteurs sociaux et économiques sont plus significatifs que l'âge, le sexe ou le comportement pour expliquer les accidents d'enfants [12].

Mais, la concentration de la pauvreté n'est pas le seul facteur explicatif : la forme urbaine du quartier de résidence joue également un rôle. Mueller et al. montrent que les enfants résidant dans des immeubles ont 5,5 fois plus de risque d'accident que les enfants habitant des maisons individuelles [23] en raison d'une exposition plus grande au risque avec des temps importants passés en dehors du logement. De plus, la forme urbaine du quartier influence les représentations spatiales des individus : à partir d'une enquête qualitative, Faure et Garnier montrent en 1994 qu'il existe une relation forte entre la structuration de la personnalité, l'autonomie et les caractéristiques de l'espace environnant (situation du quartier par rapport au centre, présence de coupures urbaines, lisibilité de l'espace, type d'accès au quartier et aménagement de ceux-ci, implantation du bâti et volumes de l'extérieur induits...). Ces auteurs concluent que les quartiers organisés pour que la voiture n'y rentre pas semblent être victimes du résultat inverse : les accidents sont nombreux en périphérie, les jeunes sont timorés et les parents anxieux interdisent aux enfants de sortir. Un réseau viaire complet et hiérarchisé facilite l'apprentissage de l'espace [7]. En effet, la pauvreté du lieu de résidence accentue les risques de catégories déjà plus impliquées (hommes, jeunes, professions intermédiaires) et détermine fortement le risque d'avoir un accident en tant que piéton. De plus, une suroccupation des logements dans le quartier de résidence, associée à une forte densité de réseau et à de forts trafics, augmente significativement les risques

\footnotetext{
${ }^{3}$ Eurostat mesure la pauvreté monétaire de manière relative : le seuil est déterminé par rapport à la distribution des niveaux de vie de l'ensemble de la population. Eurostat et les pays européens utilisent en général un seuil à $60 \%$ de la médiane des niveaux de vie. Pour rendre compte de la pauvreté, d'autres indicateurs sont possibles comme le taux de chômage, le niveau d'éducation, la part des emplois précaires, le type d'emploi occupé... Par exemple, Hasselberg et al. (2001) utilisent dans leur recherche le groupe social des individus selon l'emploi occupé.
}

d'être impliqué dans un accident en tant que piéton [8]. Les piétons représentent donc un enjeu important dans les quartiers défavorisés et l'aménagement des espaces joue un rôle sur l'amélioration de la sécurité [24].

Dans ce contexte de cumul de facteurs explicatifs associés aux inégalités socio-spatiales devant le risque d'être impliqué dans un accident en tant que piéton, comment l'action publique peut-elle se positionner?

\section{Un fonds pour réduire le nombre d'accidents dans les quartiers défavorisés}

Au Royaume-Uni comme en France, dès les années 1970 se mettent en place des partenariats d'acteurs locaux pour répondre aux problèmes de pauvreté en milieu urbain. Les années Thatcher (1979-1997) se caractérisent par la prédominance de l'angle économique et foncier pour traiter des questions urbaines. En 1997, l'arrivée au pouvoir du parti travailliste promeut de nouveaux partenariats et accorde davantage de confiance aux districts. Une politique de rénovation urbaine des quartiers en difficultés, Neighbourhood Renewal, est prescrite en 1999. L'objectif est de remettre à niveau les quartiers qui souffrent de carence par rapport à la moyenne avec le recours à l'investissement privé et un soutien aux initiatives communautaires. Les principaux acteurs sont les habitants des quartiers par le biais de leur communauté, les districts et les instances ministérielles comme la Social Exclusion Unit et la Neighbourhood Renewal Unit, ainsi que des partenariats stratégiques locaux pour coordonner les différents acteurs.

Au début des années 2000, la Social Exclusion Unit publie le rapport Making the Connections : Final Report on Transport and Social Exclusion. En se référant à une revue de littérature sur ce thème [25], ce document fait le constat que les accidents de la route touchent davantage les quartiers défavorisés et les populations en difficultés, particulièrement les piétons et les enfants en raison d'un environnement plus dangereux et d'une exposition plus grande au risque routier. Notamment, les enfants défavorisés vivent à proximité de grandes voies, jouent plus fréquemment dans la rue et sont piétons. Le manque d'accès à des aires de jeux augmente le risque.

Si bien qu'entre 2002 et 2008, le Department for Transport, équivalent britannique du Ministère des Transports, a pour objectif de réduire la concentration des accidents dans les quartiers en difficultés en débloquant 17,6 millions de livres sterling (près de 22 millions d'euros) pour les districts éligibles au Neighbourhood Renewal Fund. Il s'agit de la Neighbourhood Road Safety Initiative (NRSI). Les objectifs sont de renforcer les connaissances sur les liens entre quartiers défavorisés et sécurité des déplacements (par 
exemple : publication d'un guide à destination des districts) et de financer spécifiquement des mesures visant à réduire le nombre de tués et de blessés dans les quartiers défavorisés. Les propositions opérationnelles sont de sécuriser les traversées piétonnes, de diminuer les vitesses dans les quartiers, de mener des campagnes d'information et de créer des aires de jeux pour les enfants. Les bénéfices d'une telle politique sont profitables pour les piétons. Cette politique incite des partenariats entre les différents volets de la sécurité : répression, éducation, sensibilisation et aménagements doivent être menés de front. L'État souhaite impulser, grâce à la NRSI, une transversalité entre la rénovation urbaine et la sécurité routière.

L'article cible la gestion par la sécurité routière de ces inégalités entre les habitants d'espaces différenciés. Comment les enjeux de sécurité routière sont-ils appréhendés par les politiques de rénovation urbaine ? Quels sont les liens entre les différentes politiques sectorielles ? Alors que les inégalités socio-spatiales concernent les populations, les actions s'inscrivent spatialement par des aménagements de voies, des créations de parcs de jeux pour les enfants, des actions de sensibilisation, des contrôles et de la répression. L'objectif d'une telle politique territoriale est de réduire des inégalités sociales qui s'inscrivent spatialement. Cette approche socio-spatiale de la sécurité routière est extrêmement novatrice. La Neighbourhood Road Safety Initiative (NRSI) propose ainsi un traitement des inégalités socio-spatiales devant le risque routier par l'espace : en aménageant les quartiers, les pouvoirs publics visent à réduire les risques des habitants de ce quartier. Dans quelles mesures des actions spatiales peuvent-elles réduire des inégalités socio-spatiales ? Pour éclairer une telle question, les cas des districts du Grand Manchester bénéficiant des fonds de la Neighbourhood Road Safety Initiative sont décryptés.

\section{Analyse de la transversalité entre rénovation urbaine et sécurité routière dans le Grand Manchester}

La Neighbourhood Road Safety Initiative est une expérimentation réalisée dans quinze districts pilotes de 2002 à 2008. Parmi les quinze districts, douze se situent dans le nord-ouest de l'Angleterre dans le Grand Manchester ou à proximité. Les quinze sont évalués en 2010 par un consortium de chercheurs piloté par le centre d'études des transports du University College London. Pour comprendre la gestion des projets, nous nous sommes appuyés sur cette évaluation, ainsi que sur une analyse des documents de planification, d'entretiens réalisés auprès des spécialistes de cette question au niveau national ainsi qu'auprès de responsables locaux des Road Safety Units, et de relevés de terrain concernant les aménagements d'espaces publics.

Le Grand Manchester est une métropole de plus de 2,5 millions d'habitants au nord-ouest de l'Angleterre. Dix districts le composent, dont huit éligibles au Neighbourhood Renewal Fund. À proximité, quatre districts bénéficient également des fonds de la NRSI : Liverpool, Bradford, Blackburn with Darwen et Blackpool. La localisation des douze districts dans la région du Grand Manchester bénéficiant de la Neighbourhood Road Safety Initiative est représentée sur la carte (fig. 1).

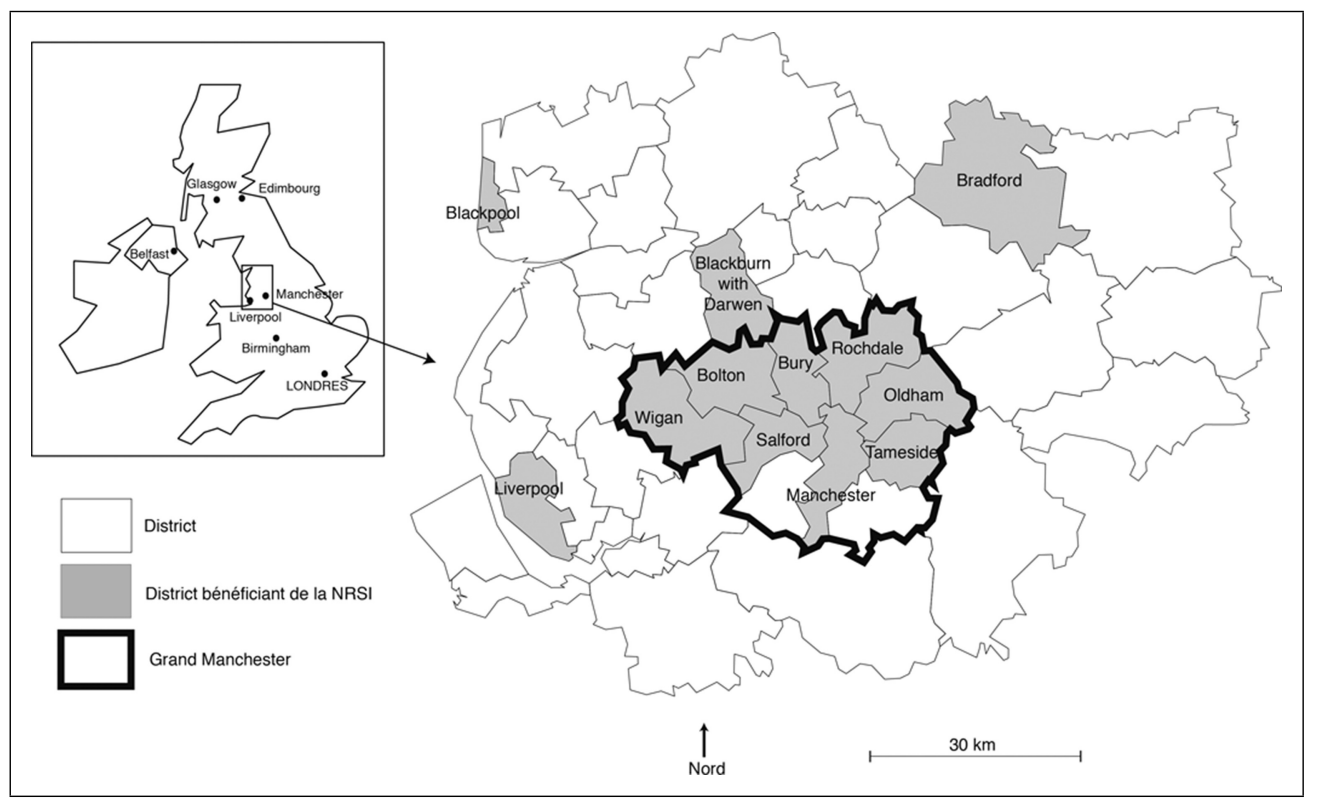

Fig. 1 Situation des districts étudiés

Source : Auteur, 2010 
Le Grand Manchester est une agglomération composée de nombreux districts défavorisés. La région a en effet connu un déclin industriel et économique dans un contexte de modernisation des processus de fabrication des textiles qui a conduit à une forte automatisation de la production, nécessitant de moins en moins de main-d'œuvre. Si bien que dans le Grand Manchester, le nombre d'emplois entre 1965 et 1995 a diminué de $24 \%$ et le taux de chômage est, en 2010, autour de $10 \%$ [26]. La région compte parmi les plus défavorisées de la Grande-Bretagne. Ainsi, Bolton fait partie des $20 \%$ des régions les plus pauvres de la Grande-Bretagne et $30 \%$ de la population n'a pas accès à un véhicule motorisé. Dans ce contexte de grande pauvreté, la majorité des districts est concernée par des actions de rénovation urbaine en étant éligible au Neighbourhood Renewal Fund.

Les projets des districts du Grand Manchester ayant bénéficié du fonds de la NRSI sont analysés. L'analyse de ces projets permet de comprendre la transversalité entre les actions de la rénovation urbaine et les enjeux de sécurité routière à l'échelle locale et territorialisée. La transversalité peut se manifester selon différents degrés d'interaction :

- une coordination entre les politiques sectorielles (degré fort d'interaction),

- une cohérence entre les objectifs poursuivis (degré moyen d'interaction),

- ou à une coopération entre les acteurs (degré faible d'interaction).

Coordonner signifie ordonner des éléments pour constituer un ensemble cohérent. Les chercheurs de l'Université Polytechnique de Lausanne utilisent ce terme dans le sens d'une structure : " les procédures de coordination renvoient à la structure, soit à la hiérarchie institutionnelle de la prise des décisions et au cadre légal dans lesquelles elles s'inscrivent, mais aussi aux stratégies déployées par les acteurs » [27]. Les auteurs distinguent le concept de coordination de celui de cohérence, renvoyant aux objectifs, et à celui de coopération, faisant référence à l'attitude des acteurs. La cohérence renvoie également à une démarche partenariale. En effet, le partenariat est un système associant des partenaires qui vise à établir des relations d'étroite collaboration. Le partenariat dans les processus de décision renvoie à une association des acteurs qui gardent leur autonomie d'action.

La méthode pour analyser la transversalité passe par une grille de lecture systématique des objectifs et des enjeux, des instruments et outils, de la conduite de projet et des représentations des acteurs techniques en charge des projets financés par la NRSI. Cette grille de lecture s'appuie sur un ensemble de matériaux :

- Les documents de cadrage à l'échelle nationale sont croisés avec des documents locaux tels que les projets de rénovation urbaine et les documents de planification. L'objectif est d'identifier la place des enjeux de sécurité dans les textes et dans les projets. Cette analyse documentaire est complétée par un diagnostic territorial à partir de relevés de terrain (aménagement des rues, mobiliers urbains...).

\section{Documents analysés :}

Documents locaux des différents districts : projets métropolitains, projets portés par la rénovation urbaine, documents de planification des transports...

AGMA, 2006, Greater Manchester Local Transport Plan 2, Manchester.

COMMUNITIES AND NEIGHBOURHOODS, 2007, The English Indices of Deprivation 2007, London, Communities and Neighbourhoods.

DEPARTMENT FOR TRANSPORT, 2000, Tomorrow's roads: safer for everyone, London, Department for Transport

DEPARTMENT FOR TRANSPORT, 2008, Road Safety Web Publication No. 7, Neighbourhood Road Safety Initiative, London, Central Team: Final, Report NRSI Central Team.

DEPARTMENT FOR TRANSPORT, 2010, Road Safety Web Publication No. 19 : Road Traffic Injury Risk in Disadvantaged Communities: Evaluation of the Neighbourood Road Safety Initiative, London, Department for Transport.

GREATER MANCHESTER TRANSPORT UNIT, 2008, Road Casualty Statistics Greater Manchester 2007, Manchester.

SOCIAL EXCLUSION UNIT, 2003, Making the Connections : Final Report on Transport and Social Exclusion

URBAN TASK FORCE, 1999, Towards an Urban Renaissance: Mission Statement.

Des entretiens semi-directifs auprès de spécialistes de cette question au niveau national (chercheurs, responsables de la NRSI...) et auprès d'acteurs locaux en charge de la sécurité routière permettent de se focaliser sur les aspects scientifiques et techniques de la mise en œuvre des politiques. L'enjeu est de comprendre l'organisation institutionnelle, les pratiques de travail et les discours des acteurs afin de comprendre l'appropriation de ces questions. 


\begin{tabular}{l}
\hline Acteurs interrogés : \\
Director of Postgraduate Taught Programmes for Faculty \\
of Health and Medical Sciences, University of Surrey \\
Greater Manchester Joint Road Safety Group \\
Chercheur à l'University College London \\
Senior Research Fellow, Faculty of Life Sciences, \\
University of the West of England \\
Principle Road Safety Officer - Bury \\
NRSI Officier, Environment \& Development Services \\
Road Safety Unit - Bury \\
Traffic Management \& Road Safety Manager - Black- \\
pool \\
Principal Road Accident Investigation Officer, Environ- \\
mental Services - Bolton \\
Head of Highway Management, Environmental \\
Services - Bolton \\
Principal Road Safety Officer, Environmental Services - \\
Bolton \\
Neighbourhood Road Safety Team - Bury \\
Traffic Management \& Road Safety Manager - Oldham \\
Road Safety Officer - Oldham
\end{tabular}

Ces matériaux sont traités selon les thématiques suivantes :

- Objectifs et enjeux des politiques : les objectifs et les enjeux de la rénovation urbaine sont analysés dans les programmes (échelle nationale) et dans les projets (échelle locale). Les objectifs donnés par l'État ont des implications très fortes sur les projets d'aménagements. Ces objectifs sont croisés avec les objectifs nationaux et locaux des politiques de sécurité routière. De cette confrontation est attendu un retour sur la cohérence entre les objectifs de la rénovation urbaine et de la sécurité routière.

- Représentations et cultures techniques : les représentations et les cultures techniques font référence à la manière dont les acteurs appréhendent la question de l'insécurité dans les quartiers et quels outils techniques sont mobilisés pour y répondre.

- Instruments et outils : les instruments des politiques de rénovation urbaine concernent les choix d'indicateurs, les procédures, les financements et les évaluations. Il s'agit de comprendre comment les instruments et les outils permettent ou non la transversalité entre les politiques sectorielles.

- Institutions et conduite de projet : l'analyse des institutions et des conduites de projets cherche à mettre en valeur la manière dont les cadres influencent la coordination entre les acteurs décisionnels techniques et les processus de décision pour la définition du projet urbain.
Cette grille de lecture transversale a pour objectif de comprendre les clés d'une politique innovante en matière de sécurité routière et de répondre au questionnement suivant : quelles sont les conditions d'application d'une gestion socio-territoriale de la sécurité routière et quelles en sont les limites?

\section{Une conduite de projet pragmatique émanant directement de l'État}

La mise en place d'un financement dédié à la réduction du nombre de tués et de blessés dans les quartiers défavorisés semble être un succès en raison d'une conduite de projet directement impulsée par l'État et de la poursuite d'objectifs précis et quantifiés.

\section{Une procédure centralisée poursuivant des objectifs chiffrés}

Le Royaume-Uni est très centralisé et fonctionne par des financements dédiés [28]. L'étude de ce procédé permet de constater l'efficacité d'un financement dédié à un objectif précis et chiffré.

$\mathrm{Au}$ Royaume-Uni, les financements pour améliorer la sécurité routière émanent directement de l'État. Pour obtenir des financements, les Road Safety Units (Unités de sécurité routière) des différentes collectivités constituent un dossier auprès du Department for Transport (équivalent $\mathrm{du}$ ministère des Transports). Pour la Neighbourhood Road Safety Initiative, la démarche est assez différente puisque le Grand Manchester est la cible de cette politique novatrice. L'objectif est de faire prendre conscience des liens entre pauvreté et sécurité routière dans une des régions urbaines les plus pauvres du Royaume-Uni et de créer de bonnes pratiques. L'obtention du fonds de la NRSI est donc plus facile à obtenir que les autres fonds d'amélioration de la sécurité routière, mais les procédures restent identiques. "C'est un financement spécial par le Department for Transport. Le Department for Transport travaille de cette façon : ils ont des financements généraux pour les Local Transport Plans, et des financements, et la NRSI en fait partie » (extrait de l'entretien mené auprès des responsables de l'unité de sécurité routière à Oldham). Cet extrait d'entretien n'est pas sans rappeler les résultats de Ménerault et al. qui montrent une forte centralité des financements au Royaume-Uni [28].

Le fonds de la Neighbourhood Road Safety Initiative a mis en place des organisations afin de faciliter le partenariat entre les acteurs et la diffusion des connaissances. Il s'agit d'une représentation de l'État sur le territoire : 
- La Central Team (équipe centrale) encourage les démarches partenariales et fait office de diffuseur de bonnes pratiques.

- Le Steering Group (groupe de pilotage) identifie les potentiels liens entre les projets, explore les liens avec d'autres initiatives, notamment le Neighbourhood Renewal, qui définit la stratégie en terme de renouvellement urbain, et développe des contacts avec le Local Strategic Partnership (LSP).

- Enfin, le Project Board (conseil de projet) fait le lien entre le Department for Transport, la Central Team et le Steering Group. Concrètement, le Project Board rapporte les projets et les évolutions de l'échelle locale à l'échelle nationale, il veille au programme de travail de l'équipe centrale et reçoit les rapports élaborés par le groupe de pilotage.

- Dans chaque district bénéficiant d'un financement de la NRSI, un coordinateur est employé pour la durée du financement. Il s'agit la plupart du temps du recrutement en interne d'une personne déjà présente.

La stratégie du renouvellement des quartiers (Neighbourhood Renewal) est gérée à l'échelle des districts par le Local Strategic Partnership (LSP). Ce groupe détermine les orientations pour les années à venir à travers le contrat local (Local Area Agreement, LAA) et élabore la stratégie de rénovation des quartiers (Neighbourhood Renewal Strategy). Au Royaume-Uni, la présence d'un Local Strategic Partnership conditionne l'octroi du Neighbourhood Renewal Fund.

La Central Team travaille avec les différents Local Strategic Partnerships pour s'assurer que la sécurité routière est intégrée à leur stratégie. Le but est de faciliter l'approche « joined up » et de permettre un traitement multi-disciplinaire de l'insécurité. Pour ce faire, la Central Team informe le Local Strategic Partnership qu'il existe des disparités face à la sécurité des déplacements au niveau national et local. En effet, les LSP ont l'habitude de traiter les questions de sécurité par des luttes contre la drogue et le comportement « anti-social ». L'enjeu est alors de persuader les LSP que la sécurité routière est un enjeu transversal (« cross cutting issue ») qui requiert une approche concertée à tous les niveaux. De plus, les LSP ont toujours abordé la question de la sécurité comme un problème à l'échelle de l'arrondissement et non à l'échelle du quartier. L'autre enjeu est donc de leur faire prendre conscience que les problèmes de sécurité routière sont territorialisés.

En raison du lien entre pauvreté et insécurité, ces objectifs sont déclinés pour les quartiers défavorisés avec un fonds dédié à la réduction du nombre d'accidents. Les acteurs techniques britanniques sont sensibilisés aux recherches sur l'insécurité dans les quartiers pauvres : « la NRSI est un projet spécifique qui vient du Department for Transport pour les quartiers défavorisés. Ils ont fait ce lien entre la pauvreté et la fréquence d'occurrence des accidents, surtout pour les enfants piétons. Les personnes ont davantage d'accidents dans leur quartier à cause de leur pauvreté. Le principal focus est fait sur les piétons » (extrait de l'entretien mené auprès des responsables du service de sécurité routière à Bolton). Ces objectifs incitent donc à traiter de la problématique de sécurité routière à l'échelle urbaine.

La démarche NRSI n'est pas seulement centralisée, elle poursuit également des objectifs chiffrés dictés par l'État et repris à l'échelle locale. Les objectifs en matière de sécurité routière chiffrés explicitement par les différents documents de planification et par la stratégie du Neighbourood Renewal du Grand Manchester. En 2000, dans Tomorrow's Roads: Safer for Everyone, le gouvernement définit de nouveaux objectifs pour la stratégie de sécurité routière. Ces objectifs sont précisément chiffrés : de 2000 à 2010, le nombre de tués ou blessés graves doit diminuer de $40 \%$ par rapport à la période 1994-1998, le nombre d'enfants tués ou blessés graves de $50 \%$ et le nombre de blessés légers de $10 \%$ [13]. Les objectifs de sécurité routière sont précisément chiffrés et ne concernent pas seulement le nombre de tués mais également les blessés. Ces objectifs nationaux sont intégralement traduits à l'échelle locale et la cohérence entre les documents de planification est respectée. Dans le Grand Manchester, le second Local Transport Plan (2006) est en vigueur lors de la recherche en 2010. Ce document a des objectifs chiffrés concernant la sécurité routière pour l'horizon 2010 : une baisse de $50 \%$ des tués et blessés graves par rapport à la période 1994-1998, une baisse de $55 \%$ des enfants tués et blessés graves pour la même période et une baisse de $30 \%$ des blessés légers pour la même période. Ces objectifs sont plus ambitieux que ceux imposés par le Department for Transport, dont les évolutions atteignent respectivement $40 \%, 50 \%$ et $10 \%$ exprimés en nombre de personnes légèrement blessées pour 100 millions de véhicule.kilomètres. En 2010, le bilan est positif avec des objectifs dépassés [13].

Le centralisme de l'État est très fort : les subventions sont octroyées par le Department for Transport aux districts et des équipes décentralisées veillent au bon déroulement des actions permises par les financements. De plus, les discours sur les inégalités socio-spatiales devant les risques d'accident sont appréhendés par les acteurs locaux et il existe une forte cohérence entre les objectifs nationaux et les objectifs locaux en matière de sécurité routière. 


\section{Une conduite de projet très segmenté}

L'analyse de la conduite de projet en Grande-Bretagne montre l'unicité des acteurs en charge d'une thématique. La sécurité routière est du ressort de la Road Safety Unit qui traite de la thématique de la sécurité routière du diagnostic à la maîtrise d'œuvre, autrement dit de l'analyse des accidents à la réalisation des travaux sur voirie. La coordination avec les actions de la rénovation urbaine ne passe pas par une coordination entre les politiques sectorielles, mais par une forte segmentation des compétences.

En effet, l'objectif du Neighbourhood Renewal est de recenser l'ensemble des financements possibles pour l'amélioration des quartiers défavorisés en termes économiques, sociaux, de santé et du cadre de vie. Le fonds du Neighbourhood Road Safety Initiative est l'un des programmes possibles. Les objectifs sont très précis et visent la réduction des tués et blessés graves dans les quartiers à travers des actions de sensibilisation, de répression et d'aménagement des espaces publics. Les Local Strategic Partnerships définissent la stratégie du Neighbourhood Renewal en élaborant des Neighbourhood Action Plans ciblés pour les quartiers les plus défavorisés $\mathrm{du}$ district. Ces documents recensent les actions possibles et leurs financements. Les actions pour améliorer la sécurité routière sont renvoyées à la responsabilité des Road Safety Units : « le transport n'est pas directement leur problème » (extrait de l'entretien mené auprès de la responsable de l'unité de sécurité routière à Blackpool). Les différentes entités sont très segmentées : chacun travaille sur un point précis à une échelle précise : «nous travaillons à un niveau plus local $[\ldots]$, on travaille sur des choses beaucoup plus précises » (extrait de l'entretien mené auprès de la responsable de l'unité de sécurité routière à Blackpool), tandis que « les LSP éclairent des grandes lignes » (extrait de l'entretien mené auprès des responsables de l'unité de sécurité routière à Oldham). Les différents acteurs et leurs compétences sont schématisés dans l'organigramme ci-après.

La compréhension de la conduite de projet ne peut faire l'impasse sur la conduite temporelle du projet. Les Road
Safety Units sont présentes à toutes les étapes du processus de décision des projets relatifs à la réduction du nombre d'accidents. Les Road Safety Units sont autonomes dans leurs décisions et financent les projets d'amélioration de la sécurité des plus vulnérables grâce aux fonds de la Neighbourhood Road Safety Initiative. La NRSI apparaît alors comme un guichet unique pour améliorer la sécurité.

Pour obtenir les financements de la Neighbourhood Road Safety Initiative, les Road Safety Units constituent un dossier pour obtenir des subventions auprès du Department for Transport. Les projets se construisent sur un diagnostic précis de l'accidentologie et proposent des pistes pour réduire le nombre d'accidents réalisé par les Road Safety Units. Les diagnostics de sécurité routière détaillent les types de collisions et analysent les regroupements géographiques d'accidents (geographical cluster). L'analyse statistique des accidents, et en particulier l'analyse des zones de concentrations d'accidents permet de prioriser les sites pour les aménagements. D'autres critères sont pris en compte pour identifier et prioriser les sites comme les enquêtes de vitesse, les enquêtes de trafic, les générateurs de trafic, la densité de logements et les facteurs socio-économiques (Greater Manchester, Local Transport Plan 2).

Intégrer les facteurs socio-économiques dans la hiérarchisation des sites prioritaires pour améliorer la sécurité routière est novateur : " il permet de se concentrer sur les quartiers défavorisés. Avant la NRSI nous ne considérions pas la pauvreté comme une possibilité de prioriser les différents sites, aujourd'hui c'est le cas. Avant, nous nous centrions surtout sur le centre de Bolton. " (extrait de l'entretien mené auprès des responsables de l'unité de sécurité routière à Bolton). À notre connaissance, il n'existe pas d'autres exemples de ce type à l'international. Cette innovation s'explique par l'évolution des dispositifs au Royaume-Uni de territorialisation de la sécurité routière. Dans les années 1980, une expérimentation de Gestion Urbaine de la Sécurité a été expérimentée dans cinq villes par le Transport Research Laboratory. Mais cette politique n'a pas clairement pu développer des stratégies d'action cohérente à l'échelle d'un quartier ou d'une ville. Si bien que le projet «Ville plus Sûre » (Safer City Project) a

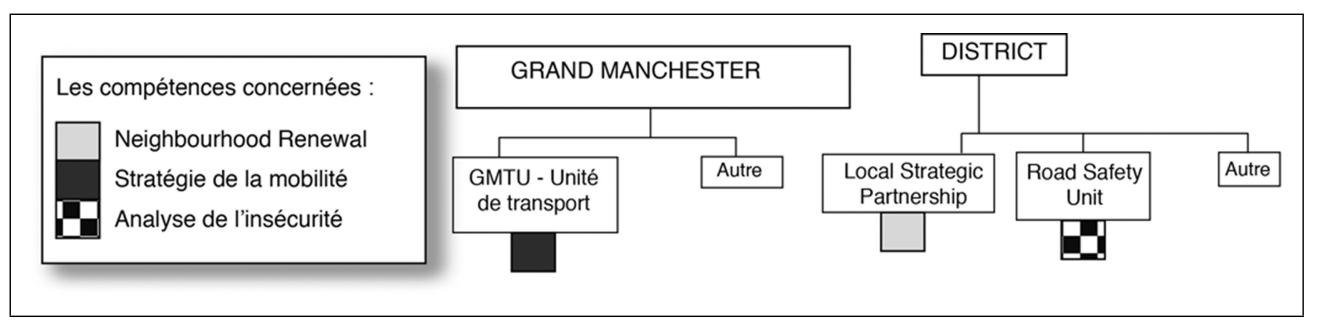

Fig. 2 Services et partage des compétences dans le Grand Manchester

Source : Auteur, 2010

Necplus 
été conduit de 1996 à 2001 dans la ville de Gloucester montrant l'influence du développement urbain sur la sécurité et donc la pertinence d'un traitement spatial de la sécurité routière.

Une fois la subvention obtenue pour un projet, les Road Safety Units mettent en œuvre le projet défini suite à l'analyse précise des accidents ayant eu lieu dans le quartier et un travail avec les communautés locales. Le fonds de la Neighbourhood Road Safety Initiative a renforcé ce travail de partenariat avec les communautés locales et entre les différents services : " c'est une différence de manière de faire. On reçoit de l'argent pour essayer, pour expérimenter. [...] alors on teste des actions en combinant par exemple dans un même espace des améliorations de traversées pour les piétons, des mesures pour sensibiliser et des actions pour éduquer » (extrait de l'entretien mené auprès des responsables de l'unité de sécurité routière à Bolton). Les mesures ne sont donc pas uniquement de l'ingénierie et de l'aménagement de voies, mais comportent également un volet important de sensibilisation. Les partenariats entre les acteurs et avec les communautés locales sont promus pour poursuivre l'objectif précis de réduction du nombre d'accidents, mais le projet est réalisé du diagnostic à la maîtrise d'œuvre par la Road Safety Unit. Cette forte segmentation des compétences conduit à un succès de la politique menée grâce au fonds de la Neighbourhood Road Safety Initiative. Toutefois, comme le montre l'évaluation de la Neighbourhood Road Safety Initiative, les partenariats, et a forciori la coordination, restent difficiles à installer.

\section{Une difficile coordination entre les politiques sectorielles}

L'évaluation de la NRSI est réalisée par une équipe pluridisciplinaire en 2010 [13]. Cette évaluation se compose de trois volets :

- une analyse quantitative des accidents qui montre une baisse significative dans les quinze districts ayant bénéficié de la NRSI,

- une analyse qualitative auprès des habitants de ces quartiers autour des déplacements, de la qualité du cadre de vie et de l'insécurité ressentie qui expose également une amélioration du ressenti des habitants des quartiers concernés,

- et le dernier volet concerne les liens entre les différents acteurs institutionnels.

Concernant ce dernier point, la Neighbourhood Road Safety Initiative propose une approche novatrice pour améliorer la sécurité. Le principal défi est de construire des partenariats entre les professionnels et les praticiens impliqués dans la santé, l'ingénierie de la sécurité routière et l'éducation pour répondre aux multiples facteurs de risque influençant les accidents. L'importance stratégique de la NRSI a été facilitée par les partenariats entre la Central Team et les coordinateurs NRSI. Ce partenariat a permis de souligner l'importance de la sécurité routière comme un problème de rénovation urbaine, qui jusqu'alors avait été souvent ignorée par les Local Strategic Partnership (LSP). Toutefois, les acteurs locaux interrogés ${ }^{4}$ dans ce rapport ont estimé qu'il $\mathrm{y}$ avait un manque de compréhension en terme de gestion de projet pour mettre en place les partenariats. Il n'y a pas de compréhension claire de ce qui est entendu par "l'innovation », « le partenariat », « le renouvellement et la rénovation », « la consultation ». Pour tous ces termes, des définitions opérationnelles auraient été nécessaires. La transversalité entre des secteurs aussi différents que sont la rénovation urbaine et la sécurité routière n'est donc pas aisée malgré les instances étatiques directement présentes sur le territoire du Grand Manchester qui œuvrent pour une coordination.

En effet, les entretiens réalisés dans le cadre de la recherche montrent que les techniciens renvoient les compétences aux services intéressés : " on ne travaille pas avec eux parce qu'ils définissent des orientations pour l'ensemble des quartiers défavorisés » (extrait de l'entretien mené auprès de la responsable de l'unité de sécurité routière à Bolton). Cette difficile transversalité se manifeste également dans les documents de planification. Si les documents relatifs à la stratégie du Neighbourhood Renewal abordent les questions de sécurité, la définition des actions et leur mise en œuvre sont intégralement traitées par les services communaux en charge de la sécurité routière. Par exemple, à Bolton, le Neighbourhood Action Plan pour le quartier de Rumworth, au sud du centre de Bolton, comporte une carte des accidents et présente les actions entreprises grâce au fonds NRSI sur la Windermere Road, axe particulièrement accidentogène. Mais le document ne traite pas davantage la question de la sécurité routière : les actions sont renvoyées à l'équipe de sécurité routière qui élabore le diagnostic du quartier en matière de sécurité routière, définit les actions et les met en œuvre. Se pose alors la question de la coordination des projets entre la rénovation urbaine et la sécurité routière dans la mesure où chaque secteur traite ses problématiques sans avoir d'impact sur les autres secteurs.

Si la NRSI n'a pas impulsé une coordination entre les politiques sectorielles, elle a véritablement conduit à une

\footnotetext{
${ }^{4}$ La liste des personnes interrogées nous a été fournie par Richard Kimberlee. Au total, 79 personnes ont été interrogées. Leurs fonctions sont très diverses : acteurs des partenariats stratégiques locaux, ingénieurs, responsables d'associations locales, policiers...
} 
meilleure coopération entre les acteurs, coopération qui perdure après la Neighbourhood Road Safety Initiative. En effet, dans son plan stratégique pour améliorer la sécurité routière, l'autorité locale de Salford citent les responsables des quartiers défavorisés comme partenaires-clés pour poursuivre l'objectif d'une réduction des accidents impliquant des enfants à l'horizon 2015 (Salford Road Safety Initiative Strategy 2011/15).

Une segmentation des compétences très précise conduit à une cohérence efficace des objectifs des politiques. Toutefois, il n'existe pas de coordination entre les acteurs traitant d'un même espace afin de construire un projet commun : les projets sont ponctuels et n'ont aucun impact sur les facteurs explicatifs de l'insécurité, comme les niveaux de trafic dans les quartiers.

\section{Des actions ciblées pour améliorer la sécurité des jeunes piétons}

Les projets financés par la NRSI sont ciblés spatialement et concernent spécifiquement les piétons et les enfants. Ces actions traduisent une politique stratégique de gestion de la sécurité routière pour réduire le nombre de tués et de blessés : les cibles sont en effet identifiées suite à un diagnostic (axes dangereux, populations vulnérables comme les enfants, etc.) et les mesures combinent plusieurs volets des facteurs de l'insécurité (aménagement de l'espace, éducation, etc.). La cohérence entre les différents volets des actions est impulsée par la démarche NRSI, notamment par les financements.

\section{Des cibles territoriales pour réduire les concentrations d'accidents}

Dans les districts du Grand Manchester, les documents relatifs à la Neighbourhood Renewal Strategy citent l'amélioration du cadre de vie et la réduction du nombre de blessés et de tués comme des priorités majeures. L'objectif principal est l'amélioration de la sécurité des piétons, particulièrement des enfants.

Ainsi à Bolton, plusieurs types d'actions sont menés : des aménagements pour améliorer la sécurité des piétons, des plans de sécurité routière près des écoles et des campagnes de sensibilisation. Une représentation spatiale des projets d'aménagement financés par le fonds de la Neighbourhood Road Safety Initiative à Bolton montre la ponctualité des actions réalisées : les projets financés par la NRSI ne concernent pas un quartier ou une zone, mais sont disséminés sur l'ensemble du territoire communal pour répondre à l'objectif d'améliorer la sécurité routière dans des endroits qui concentrent les accidents.
À Bolton, cinq projets sur sept visent à améliorer la sécurité des piétons en réduisant les vitesses, en aménageant des refuges pour les traversées ou encore en implantant des barrières le long de la voie. Ces projets ont comme objectifs de protéger les piétons du trafic. Ces actions d'aménagements se couplent avec des actions de sensibilisation (interventions auprès des communautés locales, ou panneaux pour les automobilistes) et de répression (par exemple, mise en place de caméras de surveillance à Crowpton Road).

Plus généralement sur l'ensemble des actions financées par la Neighbourhood Road Safety Initiative, les objets techniques mis en place sont des sécurisations de traversées piétonnes, des aménagements de pistes cyclables ou de cheminements piétons et la réduction des vitesses à $30 \mathrm{~km} / \mathrm{h}$ dans les rues résidentielles : « la NRSI concerne les quartiers défavorisés et les accidents correspondent à la manière dont les personnes utilisent la rue alors qu'il y a du trafic. Les projets sont donc principalement des améliorations des traversées pour les piétons, des limitations de vitesse » (extrait de l'entretien mené auprès de la responsable de l'unité de sécurité routière à Blackpool). Le type de projets financés par la NRSI montre que ce fonds vise à aménager la cohabitation du trafic et de la vie locale dans les quartiers défavorisés.

Cet enjeu d'améliorer la place du piéton se traduit également par l'aménagement de Home Zones. Deux projets parmi les dix initiatives financées par la NRSI font état d'une mise en place d'une Home Zone. Une Home Zone est aménagée dans un quartier résidentiel et se définit par une meilleure hiérarchisation du réseau, un quartier à voirie partagée avec une place importante accordée aux modes doux, une prise en compte des usagers les plus vulnérables, et des plantations végétales et aires de jeux. Les déplacements ne sont pas le seul objectif, l'amélioration $\mathrm{du}$ cadre de vie a pour but d'inverser les tendances migratoires actuelles : « La transformation des rues en des espaces d'interactions sociales est donc censée engendrer une réduction de l'exclusion sociale en conférant aux rues réaménagées en Home Zones le statut de lieu privilégié de l'inclusion sociale. Le lieu doit permettre de faire du lien social pour redonner vie aux quartiers résidentiels » [29]. Dans les quartiers pauvres, l'aménagement de rues donnant plus de droit aux piétons et aux cyclistes est donc un outil pour favoriser l'inclusion sociale dans un contexte urbain dense et fortement approprié par les habitants, notamment par les enfants.

L'ensemble des projets vise à améliorer la sécurité des piétons en réduisant les vitesses (réduction de l'espace circulé, signalisation de la présence de piétons pour les conducteurs, caméra de surveillance - Figure 4), en améliorant la visibilité (réorganisation des stationnements et des arrêts de transports en commun) et en arrêtant le trafic 


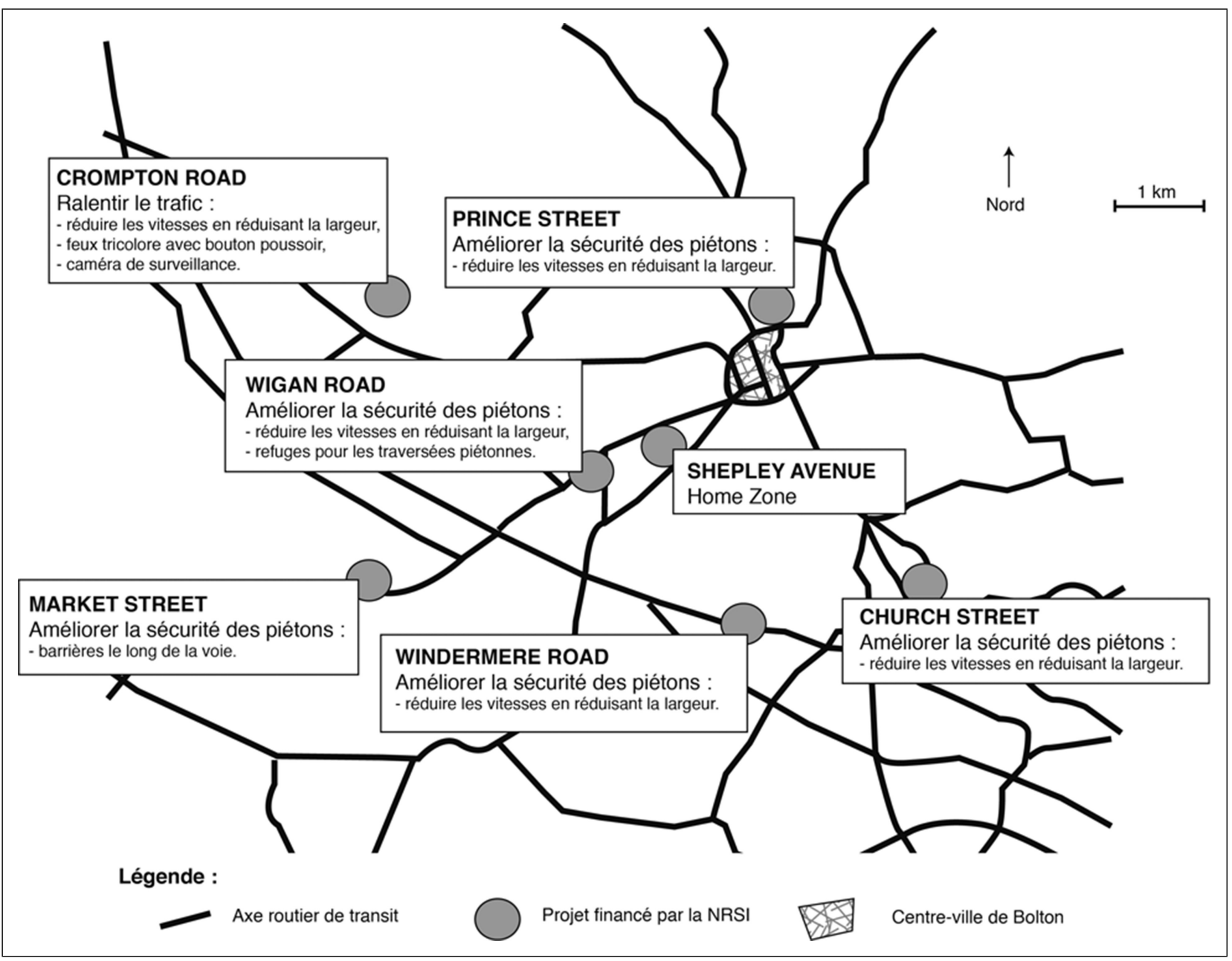

Fig. 3 Projets financés par la Neighbourhood Road Safety Initiative à Bolto

Source : Auteur, d'après les documents de planification de Bolton et les projets financés par la NRSI

pour permettre les traversées des piétons (feux tricolores, passages piétons - Figure 5). La logique principale pour réduire le nombre de tués et de blessés est donc une segmentation temporelle des usages avec des feux tricolores sur les axes à hauts niveaux de trafics pour que les piétons ou cyclistes utilisent l'espace à un autre moment.

La NRSI est une politique ciblé sur des enjeux à la fois territoriaux et socio-démographiques. En effet, au niveau socio-démographique, les cibles sont principalement les enfants piétons des communautés défavorisées, considérés comme vulnérables face au risque routier.

\section{Une priorité accordée aux enfants piétons}

Au Royaume-Uni, de nombreuses institutions s'intéressent à la sécurité des déplacements des enfants : des centres de recherches sur la santé et la sécurité des enfants comme le département de santé des enfants et des adolescents de l'université de Bristol ou le centre de la santé et du bien-être des enfants de l'université Swansea, mais aussi des associations telles que la Child Accident Prevention Trust. Les enfants sont une cible privilégiée des actions de sécurité dans la mesure où ils constituent un objectif de la politique nationale de sécurité routière.

Ces priorités se retrouvent dans le discours des acteurs techniques locaux et les responsables britanniques de la sécurité routière s'intéressent particulièrement à la sécurité des enfants dans le cadre de leur mobilité scolaire et de la forte appropriation des espaces publics comme espaces de jeux : "nous nous sommes focalisés sur les accidents, et sur les accidents ayant lieu au retour de l'école. C'est tellement dense ici qu'il y a peu de lieux pour jouer alors les enfants jouent surtout dans la rue » (extrait de l'entretien mené auprès de la responsable du service de sécurité routière à Blackpool). Ces discours sur les raisons de l'insécurité ont de forts impacts sur les choix opérés en matière de politiques publiques. La sécurité routière passe par différentes cibles. 


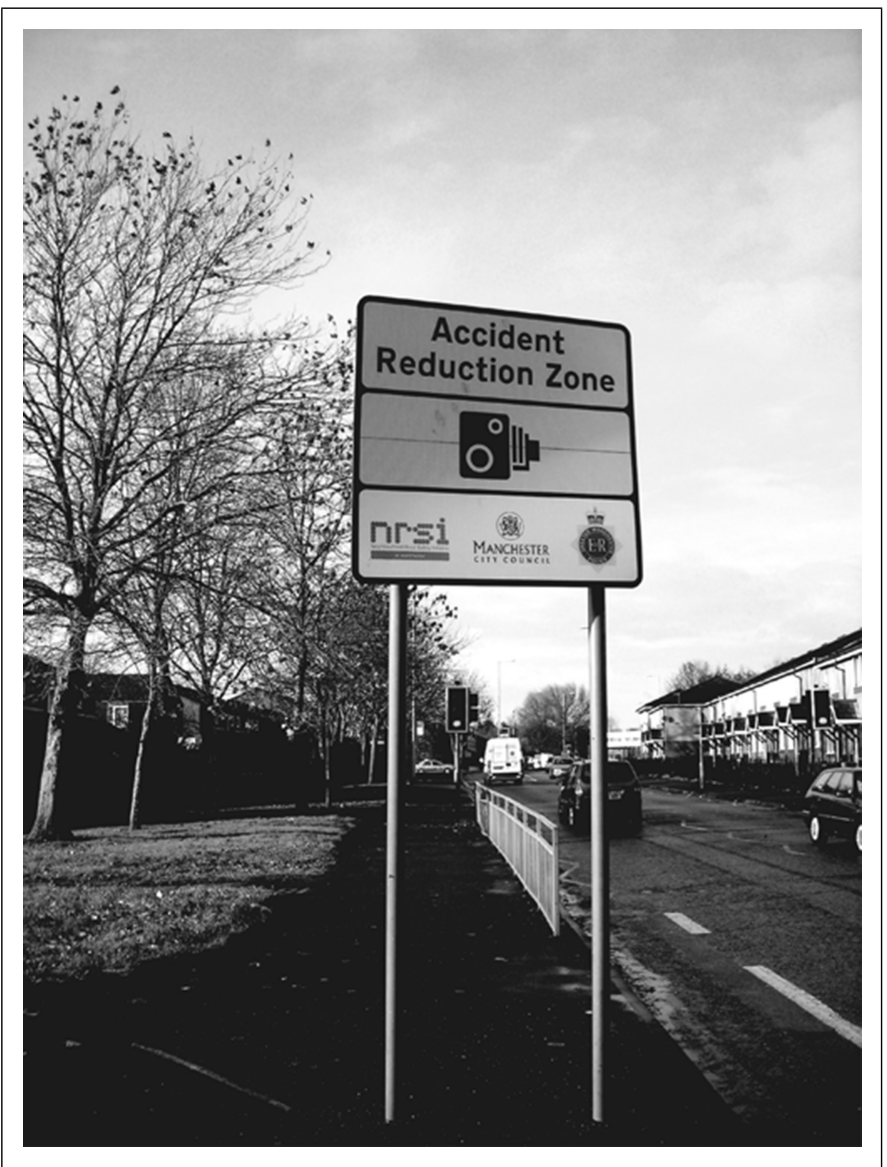

Fig. 4 Caméra de surveillance pour une réduction de vitesse, Manchester Source : Cliché personnel, 2010

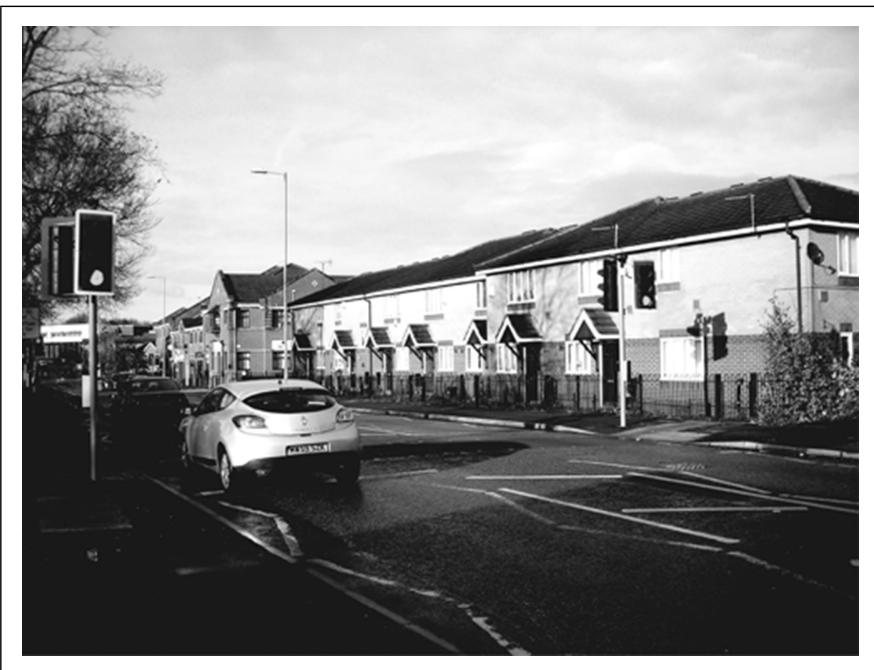

Fig. 5 Arrêt du trafic par un feu tricolore, Manchester Source : Cliché personnel, 2010

Necplus 


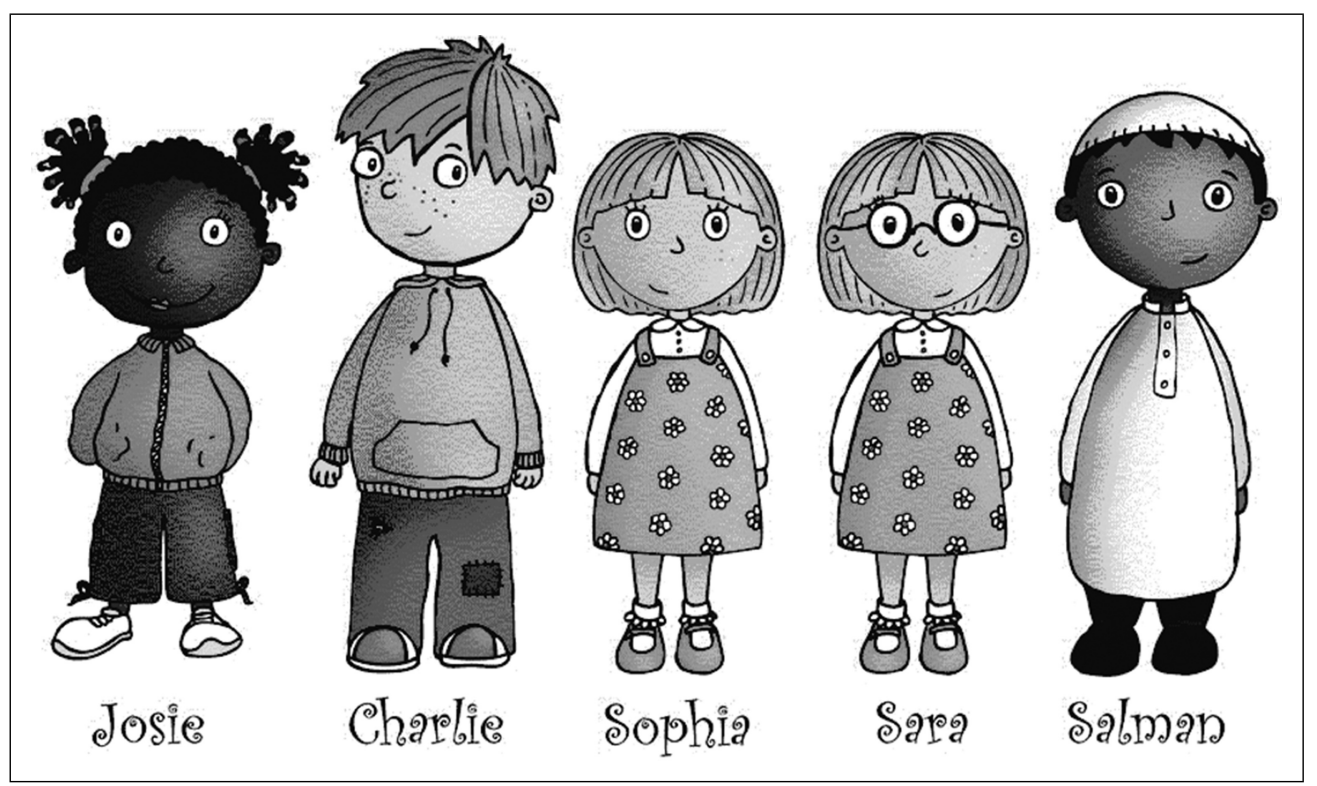

Fig. 6 Des sensibilisations pour les enfants des différentes communautés au Royaume-Uni Source : NRSI, 2010

Au Royaume-Uni, l'adaptation des espaces publics pour les enfants justifie les projets d'aménagement d'espaces publics sécurisés et la limitation des vitesses. En effet, l'enfant est, dans les représentations collectives, vulnérable et non responsable. L'entrée dans le problème de l'insécurité par les enfants permet de justifier les aménagements des espaces publics, alors qu'une entrée par les incivilités justifie davantage la mise en place d'actions de répression.

Les actions réalisées dans le cadre de la Neighbourhood Road Safety Initiative ne ciblent pas seulement les enfants, mais s'intéressent également aux différences culturelles. Au Royaume-Uni, les travaux de recherche sur les risques n'hésitent pas à cibler les communautés étrangères afin de mieux comprendre les inégalités [12,30]. Les résultats ont des impacts en terme d'action publique avec des projets sensibilisant les enfants selon leur appartenance ethnique. Des livres pour les enfants des quartiers défavorisés proposent des histoires autour de cinq enfants différents dans leur couleur de peau, qui sont présentés dans la figure 6 ci-dessus, mais également dans leurs habitudes de mobilité tant sur les motifs (Salman va à la mosquée) que sur les caractéristiques d'accompagnement (Charlie est accompagné par ses grands frères).

Les histoires visent à éduquer les enfants sur les comportements à avoir dans les espaces publics : regarder en traversant, appuyer sur le bouton-poussoir du feu tricolore et attendre avant de traverser... L'originalité de cette sensibilisation est qu'elle est en cohérence avec les aménagements réalisés (mise en place de passage piéton et feux tricolores).
Selon les acteurs techniques interrogés, une innovation impulsée par la NRSI est cette combinaison d'actions permettant d'améliorer la sécurité. Selon les chercheurs dans le domaine de la sécurité routière ayant impulsé cette initiative, ce traitement holistique de la sécurité routière n'est pas considéré comme une démarche particulièrement innovante. Ce décalage de perception de l'innovation fait état d'une différence en lien avec les pratiques des acteurs locaux : dans un contexte de forte segmentation de la conduite de projet et des responsabilités des acteurs techniques, l'intégration de différentes actions pour traiter un même enjeu semble innovant, alors que du point de vue central ces dimensions sont nécessairement à combiner.

\section{Des actions combinant différents volets pour améliorer la sécurité}

L'originalité de la Neighbourhood Road Safety Initiative est de combiner plusieurs approches pour améliorer la sécurité dans les quartiers. Des approches novatrices ont combiné des mesures « hard » concernant l'aménagement et des mesures « soft » concernant l'éducation ou la sensibilisation. Ce point est clairement mis en avant dans les textes et cité par les acteurs techniques locaux : "c'est un financement spécial. On doit donc inventer de nouvelles approches pour combiner ensemble les aménagements, l'éducation et la sensibilisation. C'est très différent des approches classiques que l'on développe avec les financements normaux qui concernent soit les aménagements, soit l'éducation, soit la sensibilisation » (extrait de l'entretien mené auprès des 


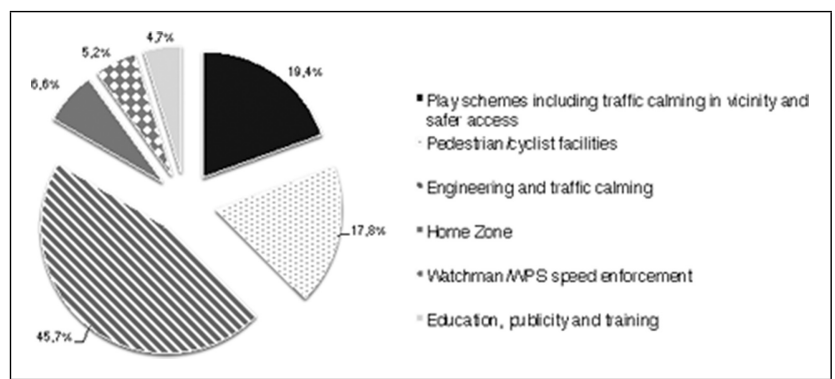

Fig. 7 Répartition du budget selon les actions pour améliorer la sécurité routière

Source : Auteur d'après NRSI, 2010

responsables de l'unité de sécurité routière à Bolton). La thématique de l'innovation a largement été abordée par l'ensemble des acteurs interrogés : "Quand la NRSI a commencé, les ingénieurs de la sécurité routière et les éducateurs de la sécurité routière ont commencé à travailler ensemble. La NRSI les a rapprochés et c'est vraiment nouveau » (extrait de l'entretien mené auprès des responsables de l'unité de sécurité routière à Oldham).

La coordination entre les actions de la rénovation urbaine et les mesures pour réduire le nombre de tués et de blessés dans les quartiers défavorisés restent difficiles. En revanche, la NRSI a permis d'encourager une véritable coopération entre les acteurs. En effet, les techniciens notent que certaines habitudes de travail demeurent suite à la mise en place de projet partenarial : « certaines habitudes de travail restent [...]. Maintenant quand on fait des aménagements, on sait que cela ne suffit pas et qu'il fait aussi beaucoup communiquer sur le projet, et consacrer de l'argent à l'éducation $[. .]$.$» (extrait de l'entretien mené auprès de$ la responsable de l'unité de sécurité routière à Blackpool). Cette approche innovante est appréciée par les techniciens des Road Safety Units : «C'est la combinaison de plusieurs approches qui fait le succès de ce fond ! » (extrait de l'entretien mené auprès des responsables de l'unité de sécurité routière à Bury).

Cette combinaison de plusieurs actions est notamment permise par le biais de financements. Une mise en graphique des différents projets financés par la Neighbourhood Road Safety Initiative permet de dégager de grandes tendances : $46 \%$ du budget sont dédiés à l'ingénierie et à la réduction des vitesses, $19 \%$ du total du budget sont alloués à l'aménagement d'aires de jeux avec des accès sécurisés, $18 \%$ du budget à des équipements pour les piétons et les cyclistes, et $7 \%$ du budget sont consacrés à l'aménagement de Home Zone (Figure ci-après).

$90 \%$ du budget total concernent donc des aménagements physiques dans les quartiers défavorisés. Ces aménagements physiques ne sont pas uniquement des mesures d'ingénierie, mais permet d'aménager des espaces de jeux pour les enfants. Toutefois, un volet est également apporté à la sensibilisation des communautés locales $(6,6 \%)$. Les budgets octroyés sont moins conséquents que pour des aménagements d'espaces en raison du moindre coût des campagnes de sensibilisation.

Malgré le fort centralisme britannique, les districts sont relativement autonomes pour le choix des types d'intervention et différents profils de districts se distinguent : celles qui se concentrent sur un type d'intervention (Blackburn), celles qui combinent deux types d'intervention (Blackpool, Bury, Rochdale, Wigan), et celles qui mixent différents types d'intervention (Bolton, Manchester, Oldham, Salford, Tameside). Les dominantes s'expliquent en partie par le corps de métier du coordinateur de la Neighbourhood Road Safety Initiative, comme le montre cet extrait d'entretien : " L'aménagement est très important à Bury, mais ce n'est pas le cas partout. Par

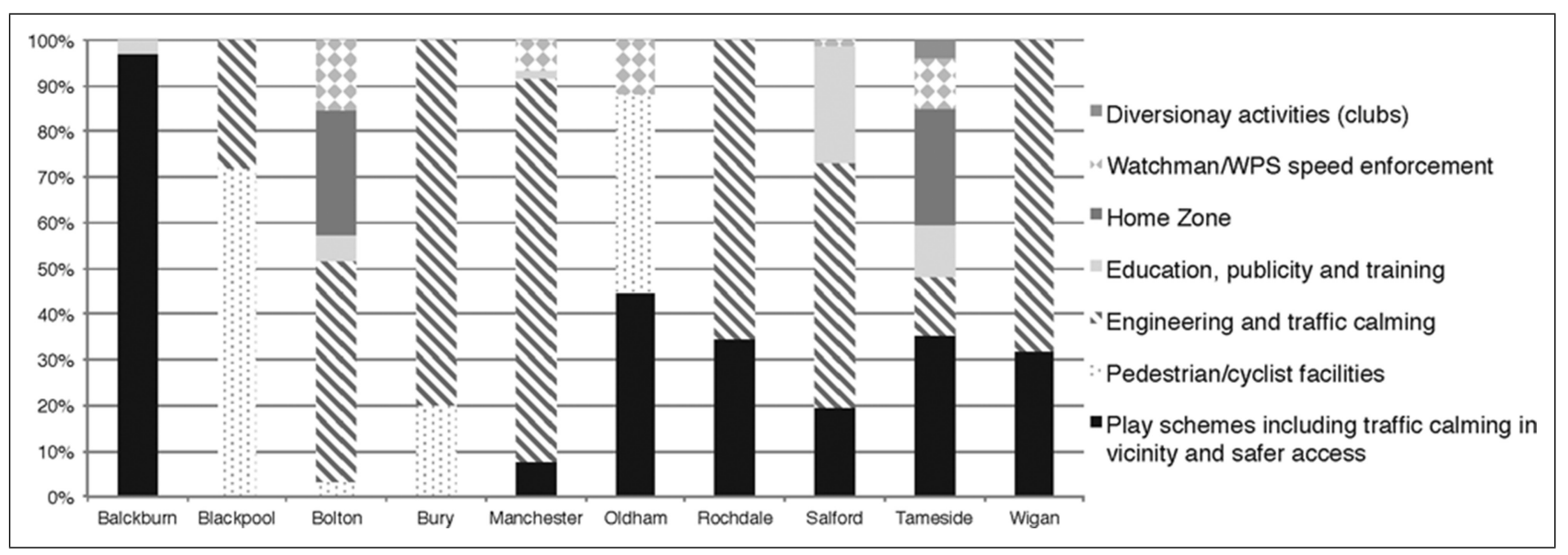

Fig. 8 Part du budget par type d'intervention et par district

Source : Auteur d'après NRSI, 2010 
exemple, à Salford les projets intègrent une dimension éducative très importante parce que la coordinatrice NRSI est sensible à ces sujets, alors qu'à Bury le coordinateur NRSI est un ingénieur » (extrait de l'entretien mené auprès des responsables de l'unité de sécurité routière à Bury).

Les financements incitent les districts à mener des actions combinatoires pour réduire le nombre de tués et de blessés : aménagement, sensibilisation, contrôle et répression sont associés dans un but d'efficacité des politiques menées. Si la coordination entre la rénovation urbaine et la sécurité routière demeure difficile à mettre en place, la NRSI aura permis une cohérence entre les objectifs des différentes politiques et une coopération entre les acteurs.

\section{Conclusion}

Au Royaume-Uni, les actions sont extrêmement segmentées. La coordination est assurée par l'État qui subventionne des projets précis et par les districts qui recensent les financements possibles dans des documents stratégiques de planification comme le Local Transport Plan ou la Neighbourhood Renewal Strategy. Les services en charge de la rédaction de ces documents stratégiques n'ont aucune compétence pour aménager les espaces : les compétences reviennent aux services concernés. Au Royaume-Uni, chaque service s'occupe de son domaine sans empiéter sur les domaines des autres. Les objets à l'interstice de plusieurs champs, comme la sécurité routière, sont donc difficilement appréhendés par ce modèle britannique : la sécurité explicite est prise en compte, mais les acteurs techniques n'ont aucune marge de manœuvre sur les processus conduisant aux situations d'insécurité comme les niveaux de trafic dans le quartier. Au Royaume-Uni, seule la rue est concernée par les aménagements pour réduire l'insécurité des déplacements : feux tricolores, passages piétons, limitation des vitesses... sont autant d'objets ponctuels utilisés au Royaume-Uni pour réduire le nombre de tués et de blessés.

$\mathrm{Si}$ le Royaume-Uni met en place des politiques très segmentées, la NRSI incite à des partenariats entre les acteurs en charge des différents volets de la sécurité : répression, sensibilisation, contrôle, aménagement... Cette initiative est considérée comme très innovante selon les acteurs techniques locaux, alors qu'elle est perçue comme indispensable à l'échelle du gouvernement. L'analyse de la transversalité entre les actions de la rénovation urbaine et les mesures pour réduire le nombre de tués et de blessés dans les quartiers défavorisés dans le Grand Manchester montre que les politiques menées en Grande-Bretagne relèvent plutôt de la cohérence des objectifs et de la coopération entre les acteurs techniques, que de la coordination des politiques. La Neighbourhood Road Safety Initiative a eu un impact fort sur la coopération entre les acteurs techniques. En effet, même si la NRSI n'a pas été reconduite après son évaluation en 2010 en raison d'évolutions financières et politiques, les acteurs techniques déclarent avoir pris l'habitude de consulter les autres acteurs, notamment ceux de la rénovation urbaine, et les documents stratégiques concernant la sécurité routière mentionnent les acteurs de la rénovation urbaine comme des partenaires-clés pour réduire le nombre d'enfants piétons tués ou blessés.

Selon les acteurs techniques, cette initiative est doublement innovante : la Neighbourhood Road Safety Initiative a renouvelé les manières d'affecter les ressources pour choisir les cibles des actions pour la sécurité routière en s'appuyant sur des mesures de la pauvreté, et elle a contribué à mener des actions combinant différents volets pour améliorer la sécurité, notamment celle des enfants piétons.

Cette approche originale semble être un succès en raison d'une forte baisse du nombre d'accidents dans les quartiers défavorisés [13]. Le Royaume-Uni a des niveaux de sécurité bien supérieurs à ceux de la France : s'inspirer des réalisations de l'outre-Manche paraît une action judicieuse.

\section{Références}

1. Offner J-M, Pumain D (eds.) (1996) Réseaux et territoires, significations croisées. Édition de l'Aube, La Tour d'Aigues, $281 \mathrm{p}$

2. Brès A (2006) De la voirie à la rue : riveraineté et attrition. Des stratégies d'inscription territoriale des mobilités périurbaines. Flux $4: 87-95$

3. Terrin J-J (eds.) (2011) Le piéton dans la ville. L'espace public protégé. Éditions Parenthèses, Marseille, $279 \mathrm{p}$

4. Edwards P, Green J, Roberts I, Grundy C, et al. (2006) Deprivation and road safety in London: A report to the London Road Safety Unit. London School of Hygiene \& Tropical Medicine, Londres, $124 \mathrm{p}$

5. Fleury D, Peytavin J-F, Alam T, et al. (2010) Inégalité sociale et Risque Routier. L'apport d'une approche territorialisée. Cahiers Scientifique du Transport 57 : 45-62

6. Haddak M, Pochet P, Licaj I, et al. (2009) Inégalités sociales et territoriales de mobilité et d'exposition au risque routier chez les jeunes - Enquêtes pilotes. Rapport intermédiaire $\mathrm{n}^{\circ} 2$ UMRESTTE-LET, Bron, $71 \mathrm{p}$

7. Faure A, Garnier F (1994) Les jeunes, les grands ensembles et la sécurité routière. Rapport de Arch'urba pour la Direction de la Recherche et des Affaires Scientifiques et Techniques, Paris, $58 \mathrm{p}$

8. Godillon S (2012) La rénovation urbaine, une opportunité de réduire les inégalités socio-spatiales d'être impliqué dans un accident dans les espaces publics, thèse de doctorat de Géographie-Aménagement, Université Paris 1 Panthéon Sorbonne, Paris, $389 \mathrm{p}$

10. Gilbert C (2003) La fabrique des risques. Cahiers internationaux de sociologie, 1(114) : 55-72

11. Kendrick D (1993) Prevention of pedestrian accidents. Archives of Disease in Childhood 68: 669-672

12. Ward H, Cave J, Morrison A, et al. (1994) Pedestrian activity and accident risk. AA Foundation for road safety research, Londres 
13. Christie N (1995) The high risk child pedestrian: socio-economic and environmental factors in their accidents. Transport Research Laboratory, Crowthorne, $33 \mathrm{p}$

14. Department for Transport (2010) Road Safety Web Publication No. 19: Road Traffic Injury Risk in Disadvantaged Communities: Evaluation of the Neighbourood Road Safety Initiative, London, Department for Transport

15. Cubbin C, Leclere FB, Smith GS (2000) Socioeconomic status and injury mortality: individual and neighbourhood determinants. Journal of Epidemiology and Community Health 54: 517-524

16. O'Campo P, Rao R, Gielen AC, et al. (2000) Injury-producing events among children inb low-income communities: the role of community caracteristics. Journal of Urban Health: Bulletin of the New-York Academy of Medecine 77: 34-39

17. Simpson K, Janssen I, Craig W. M, et al (2005) Multilevel analysis of associations between socioeconomic status and injury among Canadian adolescents. Journal of Epidemiology and Community Health 59: 1072-1077

18. Borrell C, Rodriguez M, Ferrando J, et al. (2002) Role of individual and contextual effects in injury mortality: new evidence for a small area analysis. Injury Prevention 8: 297-302

19. Abdalla I.M (1997) Statistical investigation and modelling of the relationships between road accidents and social characteristics. $\mathrm{PhD}$ de Mathématiques, Napier University, Napier, $354 \mathrm{p}$

20. Hewson P (2004) Deprived children or deprived neighbourhoods? A public health approach to the investigation of links between deprivation and injury risk with reference to child road safety in Devon County, UK. BioMed Central Public Health 4: 1-10

21. Reimers A, Laflamme L (2005) Neighbourhood social and socio-economic composition and injury risks. Acta Paediatrica 94: $1488-1494$

22. Hasselberg M, Laflamme L, Ringback Weitofbt G (2001) Socioeconomic differences in road traffic injuries during childhood and youth : a closer look at different kinds of road user. Journal of Epidemiology and Community Health 55: 858-862

23. Van Beeck E, Mackenbach JP, Looman CWN (1991) Determinants of traffic accident mortality in the Netherlands: a geographical analysis. International Journal of Epidemiology 20: 698-706.

24. Mueller BA, Rivara FP, Lii SM (1990) Environmental factors and the risk for childhood pedestrian-motor vehicle collision occurrence. American Journal of Epidemiology 132: $550-560$.

25. Fleury D (1998) Sécurité et urbanisme. La prise en compte de la sécurité routière dans l'aménagement. Presses de l'École Nationale des Ponts et Chaussées, Paris, $299 \mathrm{p}$

26. White D, Raeside R, Barker D (2000) Road Accidents and Children Living in Disadvantaged Areas: A Literature Review. Scottish Executive Central Research Unit, Edinburgh, $36 \mathrm{p}$

27. Lusso B (2010) Culture et régénération urbaine : les exemples du Grand Manchester et de la vallée de l'Emscher. Métropoles 8

28. Kaufmann V, Sager F, Ferrari Y, et al. (2003) Coordonner transports et urbanisme. Presses Polytechniques Universitaires Romandes, Lausanne, $219 \mathrm{p}$

29. Menerault P, L'Hostis A, Harman R (2007) Les transports urbains et régionaux face à un avenir incertain » In Booth $\mathrm{P}$, Breuillard $\mathrm{P}$, Fraser $\mathrm{C}$ (eds.), Aménagement et urbanisme en France et en Grande-Bretagne - Étude comparative. L'Harmattan, Paris, pp 297-320

29. Olagnier J-P (2007) Les « nouvelles » pratiques d'aménagement des rues des villes britanniques. Les Home Zones : la variante anglaise des expériences de voirie partagée. Flux 4 : 39-49

30. Agran PF, Winn DG, Andersson CL (1996) Pediatric injury hospitalization in Hispanic and non-Hispanic white children in Southern California. Archives of Pediatrics \& Adolescent Medicine 150: 400-406 